\title{
Catalytic Asymmetric Synthesis of Allylsilanes through Rhodium/Chiral Diene- Catalyzed 1,4-Addition of Alkenyl[2-(hydroxymethyl)phenyl]dimethylsilanes
}

Ryo Shintani,* Yoshitaka Ichikawa, Tamio Hayashi,* Jinshui Chen, Yoshiaki Nakao, and Tamejiro Hiyama*

Department of Chemistry, Graduate School of Science, Kyoto University, Sakyo, Kyoto 606-8502, Japan and

Department of Material Chemistry, Graduate School of Engineering, Kyoto University, Nishikyo, Kyoto 606-8510, Japan

\section{Supporting Information}

\section{General}

All air- and moisture-sensitive manipulations were carried out with standard Schlenk techniques under nitrogen or in a glove box under argon.

1,4-Dioxane was distilled over benzophenone ketyl under nitrogen. $\mathrm{MeOH}$ was distilled over $\mathrm{Mg}$ turnings under nitrogen. $\mathrm{CH}_{2} \mathrm{Cl}_{2}$ was distilled over $\mathrm{CaH}_{2}$ under nitrogen.

Tetrafluoroboric acid diethyl ether complex (Fluka), potassium fluoride (Nacalai Tesque), potassium bicarbonate (Nacalai Tesque), hydrogen peroxide (Wako Chemicals; $30 \mathrm{wt} \%$ aqueous), and titanium tetrachloride (Aldrich; $1.0 \mathrm{M}$ solution in $\mathrm{CH}_{2} \mathrm{Cl}_{2}$ ) were used as received. $\mathbf{1 a},{ }^{1} \mathbf{1 b},{ }^{1} \mathbf{1 e},{ }^{1} \mathbf{2 a},{ }^{2} \mathbf{2 b},{ }^{2} \mathbf{2 e},{ }^{2} \mathbf{2} \mathbf{f},{ }^{2}\left[\mathrm{RhCl}\left(\mathrm{C}_{2} \mathrm{H}_{4}\right)_{2}\right]_{2},{ }^{3} \mathrm{RhCl}\left(\mathrm{PPh}_{3}\right)_{3},{ }^{4}(S, S)$-Bn-bod $*{ }^{5}$ $(S, S)$-Ph-bod* ${ }^{5},(R)$-binap, ${ }^{6}(R)$-phosphoramidite, ${ }^{7}$ and $(R)$-MeO-mop ${ }^{8}$ were synthesized following the literature procedures. 1c and 1d were synthesized following the procedure for 1a. ${ }^{1} \mathbf{2} \mathbf{c}$ was synthesized following the procedure for $\mathbf{2 a}{ }^{2} \mathbf{2 d}$ was synthesized following the procedure for $2 \mathbf{e}^{2}$

All other chemicals and solvents were purchased from Aldrich, Wako Chemicals, TCI, or Kanto Chemicals and used as received.

\section{Analytical Data for New Substrates and Reagents}

(E)-1-Dimethylphenylsilyl-5-(4-methoxyphenyl)-1-penten-3-one (1c)

\footnotetext{
${ }^{1}$ Shintani, R.; Okamoto, K.; Hayashi, T. Org. Lett. 2005, 7, 4757.

${ }^{2}$ Nakao, Y.; Imanaka, H.; Sahoo, A. K.; Yada, A.; Hiyama, T. J. Am. Chem. Soc. 2005, 127, 6952.

${ }^{3}$ Cramer, R. Inorg. Synth. 1974, 15, 16.

${ }^{4}$ Osborn, J. A.; Wilkinson, G. Inorg. Synth. 1967, 10, 67.

${ }^{5}$ (a) Tokunaga, N.; Otomaru, Y.; Okamoto, K.; Ueyama, K.; Shintani, R.; Hayashi, T. J. Am. Chem. Soc. 2004, 126, 13584. (b) Otomaru, Y.; Okamoto, K.; Shintani, R.; Hayashi, T. J. Org. Chem. 2005, 70, 2503.

${ }^{6}$ Cai, D.; Payack, J. F.; Bender, D. R.; Hughes, D. L.; Verhoeven, T. R.; Reider, P. J. J. Org. Chem. 1994, 59, 7180.

${ }^{7}$ Boiteau, J.-G.; Minnaard, A. J.; Feringa, B. L. J. Org. Chem. 2003, 68, 9481.

${ }^{8}$ Uozumi, Y.; Tanahashi, A.; Lee, S.-Y.; Hayashi, T. J. Org. Chem. 1993, 58, 1945.
} 


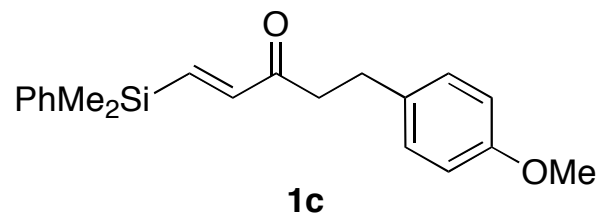

${ }^{1} \mathrm{H}$ NMR $\left(\mathrm{CDCl}_{3}\right): \delta$ 7.49-7.47 (m, 2H), 7.40-7.35 (m, 3H), $7.12\left(\mathrm{~d},{ }^{3} J_{\mathrm{HH}}=19.3 \mathrm{~Hz}, 1 \mathrm{H}\right)$, 7.13-7.09 (m, 2H), $6.81\left(\mathrm{~d},{ }^{3} J_{\mathrm{HH}}=8.7 \mathrm{~Hz}, 2 \mathrm{H}\right), 6.49\left(\mathrm{~d},{ }^{3} J_{\mathrm{HH}}=19.3 \mathrm{~Hz}, 1 \mathrm{H}\right), 3.78(\mathrm{~s}, 3 \mathrm{H})$, 2.92-2.85 (m, 4H), $0.41(\mathrm{~s}, 6 \mathrm{H}) .{ }^{13} \mathrm{C} \mathrm{NMR}\left(\mathrm{CDCl}_{3}\right): \delta 199.5,158.1,144.8,143.5,136.5$, 134.0, 133.3, 129.7, 129.4, 128.2, 114.0, 55.4, 41.5, 29.3, -3.1. Anal. Calcd for $\mathrm{C}_{20} \mathrm{H}_{24} \mathrm{O}_{2} \mathrm{Si}$ : C, 74.03; H, 7.45. Found: C, 74.13; H, 7.64.

\section{(E)-1-Dimethylphenylsilyl-4-methyl-1-penten-3-one (1d)}

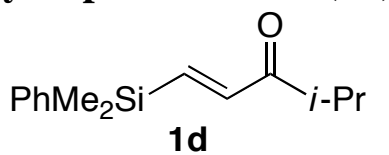

${ }^{1} \mathrm{H}$ NMR $\left(\mathrm{CDCl}_{3}\right): \delta$ 7.52-7.50 (m, 2H), 7.41-7.35 (m, 3H), $7.21\left(\mathrm{~d},{ }^{3} J_{\mathrm{HH}}=18.9 \mathrm{~Hz}, 1 \mathrm{H}\right)$, $6.58\left(\mathrm{~d},{ }^{3} J_{\mathrm{HH}}=19.0 \mathrm{~Hz}, 1 \mathrm{H}\right), 2.94\left(\mathrm{sept},{ }^{3} J_{\mathrm{HH}}=7.0 \mathrm{~Hz}, 1 \mathrm{H}\right), 1.11\left(\mathrm{~d},{ }^{3} J_{\mathrm{HH}}=6.9 \mathrm{~Hz}, 6 \mathrm{H}\right), 0.43$ $(\mathrm{s}, 6 \mathrm{H}) .{ }^{13} \mathrm{C} \mathrm{NMR}\left(\mathrm{CDCl}_{3}\right): \delta 203.5,144.4,141.9,136.7,134.0,129.6,128.1,37.9,18.5,-$ 2.9. Anal. Calcd for $\mathrm{C}_{14} \mathrm{H}_{20}$ OSi: C, 72.36; H, 8.67. Found: C, 72.60; H, 8.92.

\section{(1-Cyclohexenyl)[2-(hydroxymyethyl)phenyl]dimethylsilane (2c)}

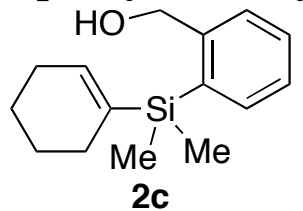

${ }^{1} \mathrm{H} \mathrm{NMR}\left(\mathrm{CDCl}_{3}\right): \delta 7.52\left(\mathrm{dd},{ }^{3} J_{\mathrm{HH}}=7.3 \mathrm{~Hz}\right.$ and $\left.{ }^{4} J_{\mathrm{HH}}=1.2 \mathrm{~Hz}, 1 \mathrm{H}\right), 7.46\left(\mathrm{dd},{ }^{3} J_{\mathrm{HH}}=7.6\right.$ $\mathrm{Hz}$ and $\left.{ }^{4} J_{\mathrm{HH}}=0.7 \mathrm{~Hz} 1 \mathrm{H}\right), 7.40\left(\mathrm{td},{ }^{3} J_{\mathrm{HH}}=7.5 \mathrm{~Hz}\right.$ and $\left.{ }^{4} J_{\mathrm{HH}}=1.4 \mathrm{~Hz}, 1 \mathrm{H}\right), 7.28\left(\mathrm{td},{ }^{3} J_{\mathrm{HH}}=7.4\right.$ $\mathrm{Hz}$ and $\left.{ }^{4} J_{\mathrm{HH}}=1.3 \mathrm{~Hz}, 1 \mathrm{H}\right), 6.13-6.10(\mathrm{~m}, 1 \mathrm{H}), 4.71\left(\mathrm{~d},{ }^{3} J_{\mathrm{HH}}=5.9 \mathrm{~Hz}, 2 \mathrm{H}\right), 2.10-2.06(\mathrm{~m}, 2 \mathrm{H})$, 2.00-1.96 (m, 2H), $1.73\left(\mathrm{t},{ }^{3} J_{\mathrm{HH}}=6.1 \mathrm{~Hz}, 1 \mathrm{H}\right) 1.64-1.56(\mathrm{~m}, 4 \mathrm{H}), 0.38(\mathrm{~s}, 6 \mathrm{H}),{ }^{13} \mathrm{C} \mathrm{NMR}$ $\left(\mathrm{CDCl}_{3}\right): \delta 146.8,138.2,138.1,136.3,135.5,129.7,128.0,127.0,65.3,27.0,26.9,23.0,22.4$, -2.1. Anal. Calcd for $\mathrm{C}_{15} \mathrm{H}_{22} \mathrm{OSi}$ : C, 73.11; H, 9.00. Found: C, 73.30; H, 9.13.

(E)-(2-Buten-2-yl)[2-(hydroxymyethyl)phenyl]dimethylsilane (2d)

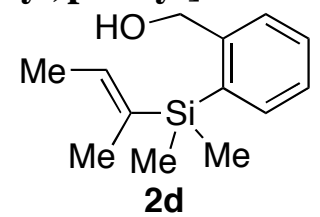

${ }^{1} \mathrm{H}$ NMR $\left(\mathrm{CDCl}_{3}\right): \delta 7.53\left(\mathrm{dd},{ }^{3} J_{\mathrm{HH}}=7.3 \mathrm{~Hz}\right.$ and $\left.{ }^{4} J_{\mathrm{HH}}=1.0 \mathrm{~Hz}, 1 \mathrm{H}\right), 7.46\left(\mathrm{dd},{ }^{3} J_{\mathrm{HH}}=7.5\right.$ $\mathrm{Hz}$ and $\left.{ }^{4} J_{\mathrm{HH}}=1.2 \mathrm{~Hz}, 1 \mathrm{H}\right), 7.40\left(\mathrm{td},{ }^{3} J_{\mathrm{HH}}=7.5 \mathrm{~Hz}\right.$ and $\left.{ }^{3} J_{\mathrm{HH}}=1.3 \mathrm{~Hz}, 1 \mathrm{H}\right), 7.28\left(\mathrm{td},{ }^{3} J_{\mathrm{HH}}=7.4\right.$ $\mathrm{Hz}$ and $\left.{ }^{4} J_{\mathrm{HH}}=1.3 \mathrm{~Hz}, 1 \mathrm{H}\right), 5.95\left(\mathrm{qq},{ }^{3} J_{\mathrm{HH}}=6.5 \mathrm{~Hz}\right.$ and $\left.{ }^{4} J_{\mathrm{HH}}=1.7 \mathrm{~Hz}, 1 \mathrm{H}\right), 4.69\left(\mathrm{~d},{ }^{3} J_{\mathrm{HH}}=\right.$ $6.1 \mathrm{~Hz}, 2 \mathrm{H}), 1.71\left(\mathrm{dq},{ }^{3} J_{\mathrm{HH}}=6.5 \mathrm{~Hz}\right.$ and $\left.{ }^{5} J_{\mathrm{HH}}=1.0 \mathrm{~Hz}, 3 \mathrm{H}\right), 1.66\left(\mathrm{t},{ }^{3} J_{\mathrm{HH}}=6.3 \mathrm{~Hz}, 1 \mathrm{H}\right), 1.66-$ $1.64(\mathrm{~m}, 3 \mathrm{H}), 0.39(\mathrm{~s}, 6 \mathrm{H}) .{ }^{13} \mathrm{C} \mathrm{NMR}\left(\mathrm{CDCl}_{3}\right): \delta 146.8,136.4,136.3,135.9,135.6,129.7$, 128.0, 127.0, 65.3, 14.52, 14.51, -1.9. Anal. Calcd for $\mathrm{C}_{13} \mathrm{H}_{20} \mathrm{OSi}$ : C, 70.85; H, 9.15. Found: C, $71.15 ; \mathrm{H}, 9.37$. 


\section{Catalytic Reactions}

\section{General Procedure for Table 2.}

A solution of $\left[\mathrm{RhCl}\left(\mathrm{C}_{2} \mathrm{H}_{4}\right)_{2}\right]_{2}(2.0 \mathrm{mg}, 10 \mu \mathrm{mol} \mathrm{Rh})$ and $(S, S)$-Ph-bod* $(2.9 \mathrm{mg}, 11 \mu \mathrm{mol})$ in 1,4-dioxane $(1.0 \mathrm{~mL})$ was stirred for $5 \mathrm{~min}$ at room temperature. $\mathrm{KOH}(12 \mu \mathrm{L}, 12 \mu \mathrm{mol}$; $1.0 \mathrm{M}$ in $\mathrm{H}_{2} \mathrm{O}$ ) was added to it and the mixture was stirred for additional $5 \mathrm{~min}$ at room temperature. Substrate $1(0.20 \mathrm{mmol})$ and nucleophile $2(0.40 \mathrm{mmol})$ were then added to it and the resulting mixture was stirred for $12 \mathrm{~h}$ at $50{ }^{\circ} \mathrm{C}$. The reaction mixture was directly passed through a pad of silica gel with EtOAc and the solvent was removed under vacuum. The residue was chromatographed on silica gel to afford 1,4-adduct 3 .

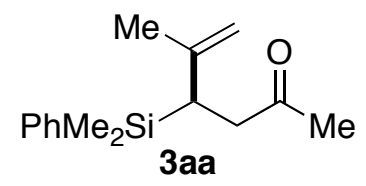

Entry 1. Colorless oil. $96 \%$ yield. The ee was determined on a Daicel Chiralpak AS column with hexane : 2-propanol $=98: 2$, flow $=0.5 \mathrm{~mL} / \mathrm{min}$. Retention times: $13.9 \mathrm{~min}$ $\left[(R)\right.$-enantiomer], 17.4 min $\left[(S)\right.$-enantiomer]. $93 \%$ ee. $[\alpha]^{20}{ }_{\mathrm{D}}+84.0\left(c 1.02, \mathrm{CHCl}_{3}\right)$. The abolute configuration was assigned by analogy with entry 5 .

${ }^{1} \mathrm{H}$ NMR $\left(\mathrm{CDCl}_{3}\right): \delta$ 7.51-7.47 (m, 2H), 7.38-7.33 (m, 3H), 4.73-4.71 (m, 1H), $4.45(\mathrm{~s}$, $1 \mathrm{H}), 2.68\left(\mathrm{dd},{ }^{2} J_{\mathrm{HH}}=16.7 \mathrm{~Hz}\right.$ and $\left.{ }^{3} J_{\mathrm{HH}}=11.7 \mathrm{~Hz}, 1 \mathrm{H}\right), 2.39\left(\mathrm{dd},{ }^{2} J_{\mathrm{HH}}=16.7 \mathrm{~Hz}\right.$ and ${ }^{3} J_{\mathrm{HH}}=$ $3.9 \mathrm{~Hz}, 1 \mathrm{H}), 2.27\left(\mathrm{dd},{ }^{3} J_{\mathrm{HH}}=11.8\right.$ and $\left.3.8 \mathrm{~Hz}, 1 \mathrm{H}\right), 2.03(\mathrm{~s}, 3 \mathrm{H}), 1.63(\mathrm{~s}, 3 \mathrm{H}), 0.32(\mathrm{~s}, 3 \mathrm{H})$, $0.31(\mathrm{~s}, 3 \mathrm{H}) .{ }^{13} \mathrm{C} \mathrm{NMR}\left(\mathrm{CDCl}_{3}\right): \delta 208.5,146.6,137.5,134.1,129.4,127.9,108.8,43.9,32.2$, 30.0, 25.0, -3.8, -4.8. Anal. Calcd for $\mathrm{C}_{15} \mathrm{H}_{22} \mathrm{OSi}$ : C, 73.11; H, 9.00. Found: C, 73.40; H, 9.03 .<smiles>C=C(C)C(CC(C)=O)[AsH2]C(C)(C)C</smiles>

Entry 2. The reaction was conducted with $(S, S)$-Bn-bod* as a ligand. Colorless oil. $95 \%$ yield. The ee was determined on a Daicel Chiralcel OD-H column with hexane : 2-propanol $=300: 1$, flow $=0.35 \mathrm{~mL} / \mathrm{min}$. Retention times: $43.9 \mathrm{~min}[(S)$-enantiomer $], 46.7 \mathrm{~min}[(R)$ enantiomer]. $97 \%$ ee. $[\alpha]^{20}{ }_{\mathrm{D}}+68.3\left(c 0.80, \mathrm{CHCl}_{3}\right)$. The abolute configuration was assigned by analogy with entry 5 .

${ }^{1} \mathrm{H}$ NMR $\left(\mathrm{CDCl}_{3}\right): \delta 4.71-4.69(\mathrm{~m}, 1 \mathrm{H}), 4.56-4.54(\mathrm{~m}, 1 \mathrm{H}), 2.73\left(\mathrm{dd},{ }^{2} J_{\mathrm{HH}}=16.1 \mathrm{~Hz}\right.$ and $\left.{ }^{3} J_{\mathrm{HH}}=12.8 \mathrm{~Hz}, 1 \mathrm{H}\right), 2.45\left(\mathrm{dd},{ }^{2} J_{\mathrm{HH}}=16.1 \mathrm{~Hz}\right.$ and $\left.{ }^{3} J_{\mathrm{HH}}=3.3 \mathrm{~Hz}, 1 \mathrm{H}\right), 2.24\left(\mathrm{dd},{ }^{3} J_{\mathrm{HH}}=12.7\right.$ and $3.2 \mathrm{~Hz}, 1 \mathrm{H}), 2.11(\mathrm{~s}, 3 \mathrm{H}), 1.73(\mathrm{~s}, 3 \mathrm{H}), 0.92(\mathrm{~s}, 9 \mathrm{H}), 0.00(\mathrm{~s}, 3 \mathrm{H}),-0.03(\mathrm{~s}, 3 \mathrm{H}) .{ }^{13} \mathrm{C}$ $\operatorname{NMR}\left(\mathrm{CDCl}_{3}\right): \delta 208.8,147.5,109.3,44.8,29.9,29.8,27.4,27.1,24.3,-6.3,-7.0$. Anal. Calcd for $\mathrm{C}_{13} \mathrm{H}_{26} \mathrm{OSi}$ : C, 68.96; H, 11.57. Found: C, 69.12; H, 11.31.

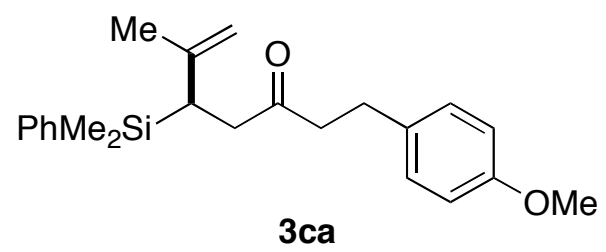

Entry 3. Colorless oil. $91 \%$ yield. The ee was determined on a Daicel Chiralpak AD-H column with hexane : 2-propanol $=300: 1$, flow $=0.5 \mathrm{~mL} / \mathrm{min}$. Retention times: $37.7 \mathrm{~min}$ $\left[(S)\right.$-enantiomer], 67.6 min $[(R)$-enantiomer $] .92 \%$ ee. $[\alpha]_{\mathrm{D}}^{20}+105\left(c 0.95, \mathrm{CHCl}_{3}\right)$. The 
abolute configuration was assigned by analogy with entry 5 .

${ }^{1} \mathrm{H} \mathrm{NMR}\left(\mathrm{CDCl}_{3}\right): \delta$ 7.49-7.47 $(\mathrm{m}, 2 \mathrm{H}), 7.38-7.33(\mathrm{~m}, 3 \mathrm{H}), 7.04\left(\mathrm{~d},{ }^{3} J_{\mathrm{HH}}=8.7 \mathrm{~Hz}, 2 \mathrm{H}\right)$, $6.79\left(\mathrm{~d},{ }^{3} J_{\mathrm{HH}}=8.5 \mathrm{~Hz}, 2 \mathrm{H}\right), 4.72-4.69(\mathrm{~m}, 1 \mathrm{H}), 4.42(\mathrm{~s}, 1 \mathrm{H}), 3.77(\mathrm{~s}, 3 \mathrm{H}), 2.79-2.71(\mathrm{~m}, 2 \mathrm{H})$, $2.65\left(\mathrm{dd},{ }^{2} J_{\mathrm{HH}}=16.7 \mathrm{~Hz}\right.$ and $\left.{ }^{3} J_{\mathrm{HH}}=11.6 \mathrm{~Hz}, 1 \mathrm{H}\right), 2.61-2.52(\mathrm{~m}, 2 \mathrm{H}), 2.34\left(\mathrm{dd},{ }^{2} J_{\mathrm{HH}}=16.7\right.$ $\mathrm{Hz}$ and $\left.{ }^{3} J_{\mathrm{HH}}=3.9 \mathrm{~Hz}, 1 \mathrm{H}\right), 2.28\left(\mathrm{dd},{ }^{3} J_{\mathrm{HH}}=11.5\right.$ and $\left.3.9 \mathrm{~Hz}, 1 \mathrm{H}\right), 1.62(\mathrm{~s}, 3 \mathrm{H}), 0.31(\mathrm{~s}, 3 \mathrm{H})$, 0.29 (s, 3H). ${ }^{13} \mathrm{C} \mathrm{NMR}\left(\mathrm{CDCl}_{3}\right): \delta 209.6,158.0,146.7,137.4,134.1,133.3,129.33,129.29$, 127.9, 113.9, 108.6, 55.3, 44.6, 43.2, 31.9, 28.8, 25.1, -3.8, -4.9. Anal. Calcd for $\mathrm{C}_{23} \mathrm{H}_{30} \mathrm{O}_{2} \mathrm{Si}$ : C, 75.36; H, 8.25. Found: C, 75.46; H, 8.19.

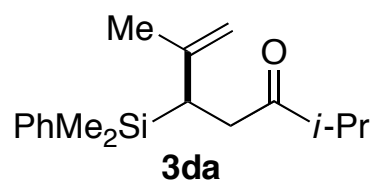

Entry 4. Colorless oil. $95 \%$ yield. The ee was determined on a Daicel Chiralpak AS column with hexane : 2-propanol $=500: 1$, flow $=0.25 \mathrm{~mL} / \mathrm{min}$. Retention times: $40.3 \mathrm{~min}$ $\left[(S)\right.$-enantiomer], 59.5 min $\left[(R)\right.$-enantiomer]. $97 \%$ ee. $[\alpha]^{20}{ }_{\mathrm{D}}+67.4\left(c 0.59, \mathrm{CHCl}_{3}\right)$. The abolute configuration was assigned by analogy with entry 5 .

${ }^{1} \mathrm{H}$ NMR $\left(\mathrm{CDCl}_{3}\right): \delta$ 7.53-7.46 $(\mathrm{m}, 2 \mathrm{H}), 7.38-7.32(\mathrm{~m}, 3 \mathrm{H}), 4.69(\mathrm{~s}, 1 \mathrm{H}), 4.41(\mathrm{~s}, 1 \mathrm{H})$, $2.75\left(\mathrm{dd},{ }^{2} J_{\mathrm{HH}}=17.3 \mathrm{~Hz}\right.$ and $\left.{ }^{3} J_{\mathrm{HH}}=11.3 \mathrm{~Hz}, 1 \mathrm{H}\right), 2.50\left(\mathrm{sept},{ }^{3} J_{\mathrm{HH}}=6.9 \mathrm{~Hz}, 1 \mathrm{H}\right), 2.42(\mathrm{dd}$, ${ }^{2} J_{\mathrm{HH}}=17.3 \mathrm{~Hz}$ and $\left.{ }^{3} J_{\mathrm{HH}}=3.8 \mathrm{~Hz}, 1 \mathrm{H}\right), 2.30\left(\mathrm{dd},{ }^{3} J_{\mathrm{HH}}=11.5\right.$ and $\left.3.7 \mathrm{~Hz}, 1 \mathrm{H}\right), 1.64(\mathrm{~s}, 3 \mathrm{H})$, $0.99\left(\mathrm{~d},{ }^{3} J_{\mathrm{HH}}=6.9 \mathrm{~Hz}, 3 \mathrm{H}\right), 0.98\left(\mathrm{~d},{ }^{3} J_{\mathrm{HH}}=6.9 \mathrm{~Hz}, 3 \mathrm{H}\right), 0.32(\mathrm{~s}, 3 \mathrm{H}), 0.30(\mathrm{~s}, 3 \mathrm{H}) .{ }^{13} \mathrm{C} \mathrm{NMR}$ $\left(\mathrm{CDCl}_{3}\right): \delta 213.9,147.3,137.6,134.1,129.3,127.9,108.2,41.0,40.5,31.2,25.4,18.4,18.2$, -3.7, -4.8. Anal. Calcd for $\mathrm{C}_{17} \mathrm{H}_{26} \mathrm{OSi}$ : C, 74.39; H, 9.55. Found: C, 74.29; H, 9.67.

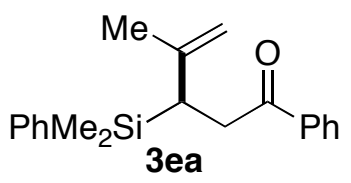

Entry 5. The reaction was conducted at $60{ }^{\circ} \mathrm{C}$. Colorless oil. $88 \%$ yield. The ee was determined on a Daicel Chiralpak AD-H column with hexane : 2-propanol $=300: 1$, flow $=$ $0.35 \mathrm{~mL} / \mathrm{min}$. Retention times: $57.1 \mathrm{~min}[(S)$-enantiomer], $59.8 \mathrm{~min}[(R)$-enantiomer]. $93 \%$ ee. $[\alpha]^{20}{ }_{\mathrm{D}}+69.1\left(c 1.00, \mathrm{CHCl}_{3}\right)$. The abolute configuration was determined by comparison of the optical rotation with that reported in the literature ${ }^{9}$ after converting it to 3-hydroxy-4methyl-1-phenylpentan-1-one (5).

${ }^{1} \mathrm{H} \mathrm{NMR}\left(\mathrm{CDCl}_{3}\right): \delta 7.81\left(\mathrm{~d},{ }^{3} \mathrm{~J}_{\mathrm{HH}}=8.2 \mathrm{~Hz}, 2 \mathrm{H}\right), 7.57-7.54(\mathrm{~m}, 2 \mathrm{H}), 7.51\left(\mathrm{t},{ }^{3} J_{\mathrm{HH}}=7.4 \mathrm{~Hz}\right.$, $1 \mathrm{H}), 7.40\left(\mathrm{t},{ }^{3} J_{\mathrm{HH}}=7.6 \mathrm{~Hz}, 2 \mathrm{H}\right), 7.39-7.35(\mathrm{~m}, 3 \mathrm{H}), 4.71-4.69(\mathrm{~m}, 1 \mathrm{H}), 4.46(\mathrm{~s}, 1 \mathrm{H}), 3.25(\mathrm{dd}$, ${ }^{2} J_{\mathrm{HH}}=16.9 \mathrm{~Hz}$ and $\left.{ }^{3} J_{\mathrm{HH}}=11.3 \mathrm{~Hz}, 1 \mathrm{H}\right), 2.94\left(\mathrm{dd},{ }^{2} J_{\mathrm{HH}}=16.9 \mathrm{~Hz}\right.$ and $\left.{ }^{3} J_{\mathrm{HH}}=3.6 \mathrm{~Hz}, 1 \mathrm{H}\right)$, $2.45\left(\mathrm{dd},{ }^{3} J_{\mathrm{HH}}=11.3\right.$ and $\left.3.6 \mathrm{~Hz}, 1 \mathrm{H}\right), 1.67(\mathrm{~s}, 3 \mathrm{H}), 0.39(\mathrm{~s}, 3 \mathrm{H}), 0.35(\mathrm{~s}, 3 \mathrm{H}) .{ }^{13} \mathrm{C}$ NMR $\left(\mathrm{CDCl}_{3}\right): \delta 199.6,146.7,137.6,137.3,134.1,132.8,129.3,128.6,128.0,127.9,108.7,38.7$, 32.0, 25.0, -3.7, -4.8. Anal. Calcd for $\mathrm{C}_{20} \mathrm{H}_{24} \mathrm{OSi}$ : C, 77.87; H, 7.84. Found: C, 78.14; $\mathrm{H}$, 7.82 .

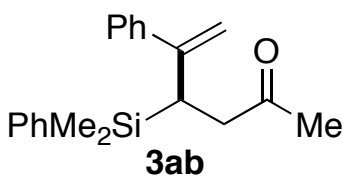

Entry 6. The reaction was conducted with $(S, S)$-Bn-bod* as a ligand. Colorless oil. $96 \%$

\footnotetext{
${ }^{9}$ Trost, B. M.; Ito, H. J. Am. Chem. Soc. 2000, 122, 12003.
} 
yield. The ee was determined on a Daicel Chiralcel OD-H column with hexane : 2-propanol $=100: 1$, flow $=0.5 \mathrm{~mL} / \mathrm{min}$. Retention times: $19.6 \mathrm{~min}[(R)$-enantiomer $], 25.1 \mathrm{~min}[(S)$ enantiomer]. $93 \%$ ee. $[\alpha]^{20}{ }_{\mathrm{D}}+197\left(c 1.01, \mathrm{CHCl}_{3}\right)$. The absolute configuration was assigned by analogy with entry 5 .

${ }^{1} \mathrm{H} \mathrm{NMR}\left(\mathrm{CDCl}_{3}\right): \delta 7.48\left(\mathrm{~d},{ }^{3} J_{\mathrm{HH}}=7.0 \mathrm{~Hz}, 2 \mathrm{H}\right), 7.44-7.41(\mathrm{~m}, 2 \mathrm{H}), 7.36-7.27(\mathrm{~m}, 5 \mathrm{H})$, $7.24\left(\mathrm{tt},{ }^{3} J_{\mathrm{HH}}=7.2 \mathrm{~Hz}\right.$ and $\left.{ }^{4} J_{\mathrm{HH}}=1.4 \mathrm{~Hz}, 1 \mathrm{H}\right), 5.15(\mathrm{~s}, 1 \mathrm{H}), 4.66(\mathrm{~s}, 1 \mathrm{H}), 2.98\left(\mathrm{dd},{ }^{3} J_{\mathrm{HH}}=\right.$ 11.4 and $3.8 \mathrm{~Hz}, 1 \mathrm{H}), 2.86\left(\mathrm{dd},{ }^{2} J_{\mathrm{HH}}=17.0 \mathrm{~Hz}\right.$ and $\left.{ }^{3} J_{\mathrm{HH}}=11.4 \mathrm{~Hz}, 1 \mathrm{H}\right), 2.49\left(\mathrm{dd},{ }^{2} J_{\mathrm{HH}}=17.0\right.$ $\mathrm{Hz}$ and $\left.{ }^{3} J_{\mathrm{HH}}=3.8 \mathrm{~Hz}, 1 \mathrm{H}\right), 2.07(\mathrm{~s}, 3 \mathrm{H}), 0.20(\mathrm{~s}, 3 \mathrm{H}), 0.06(\mathrm{~s}, 3 \mathrm{H}) .{ }^{13} \mathrm{C} \mathrm{NMR}\left(\mathrm{CDCl}_{3}\right): \delta$ 208.3, 151.1, 144.2, 137.1, 134.1, 129.3, 128.3, 127.8, 127.5, 127.1, 110.0, 44.3, 30.6, 28.8, 3.3, -5.3. Anal. Calcd for $\mathrm{C}_{20} \mathrm{H}_{24} \mathrm{OSi}$ : C, 77.87; H, 7.84. Found: C, 77.73; H, 7.72.

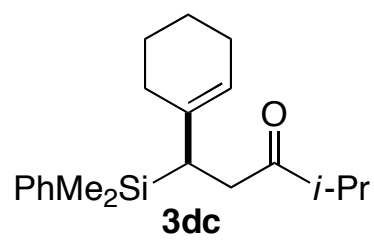

Entry 7. Colorless oil. $90 \%$ yield. The ee was determined on a Daicel Chiralpak AS column with hexane : 2-propanol $=500: 1$, flow $=0.25 \mathrm{~mL} / \mathrm{min}$. Retention times: 19.9 min $\left[(S)\right.$-enantiomer], 28.3 min $\left[(R)\right.$-enantiomer]. $92 \%$ ee. $[\alpha]^{20}{ }_{\mathrm{D}}+40.7\left(c 1.01, \mathrm{CHCl}_{3}\right)$. The absolute configuration was assigned by analogy with entry 5 .

${ }^{1} \mathrm{H}$ NMR $\left(\mathrm{CDCl}_{3}\right): \delta$ 7.50-7.47 (m, 2H), 7.36-7.32 (m, 3H), 5.18-5.15 (m, 1H), $2.72(\mathrm{dd}$, ${ }^{2} J_{\mathrm{HH}}=16.9 \mathrm{~Hz}$ and $\left.{ }^{3} J_{\mathrm{HH}}=11.1 \mathrm{~Hz}, 1 \mathrm{H}\right), 2.51\left(\mathrm{sept},{ }^{3} J_{\mathrm{HH}}=7.0 \mathrm{~Hz}, 1 \mathrm{H}\right), 2.37\left(\mathrm{dd},{ }^{2} J_{\mathrm{HH}}=16.8\right.$ $\mathrm{Hz}$ and $\left.{ }^{3} J_{\mathrm{HH}}=4.1 \mathrm{~Hz}, 1 \mathrm{H}\right), 2.12\left(\mathrm{dd},{ }^{3} J_{\mathrm{HH}}=11.2\right.$ and $\left.4.2 \mathrm{~Hz}, 1 \mathrm{H}\right), 1.98-1.91(\mathrm{~m}, 2 \mathrm{H}), 1.90-$ $1.82(\mathrm{~m}, 1 \mathrm{H}), 1.73-1.66(\mathrm{~m}, 1 \mathrm{H}), 1.52-1.46(\mathrm{~m}, 2 \mathrm{H}), 1.45-1.39(\mathrm{~m}, 2 \mathrm{H}), 0.983\left(\mathrm{~d},{ }^{3} J_{\mathrm{HH}}=7.1\right.$ $\mathrm{Hz}, 3 \mathrm{H}), 0.978\left(\mathrm{~d},{ }^{3} J_{\mathrm{HH}}=6.9 \mathrm{~Hz}, 3 \mathrm{H}\right), 0.30(\mathrm{~s}, 3 \mathrm{H}), 0.27(\mathrm{~s}, 3 \mathrm{H}) .{ }^{13} \mathrm{C} \mathrm{NMR}\left(\mathrm{CDCl}_{3}\right): \delta 214.6$, 138.5, 138.1, 134.2, 129.2, 127.8, 120.2, 40.7, 40.6, 31.1, 31.0, 25.5, 23.3, 22.7, 18.4, 18.1, 3.6, -4.4. Anal. Calcd for $\mathrm{C}_{20} \mathrm{H}_{30} \mathrm{OSi}$ : C, 76.37; H, 9.61. Found: C, 76.35; H, 9.61.

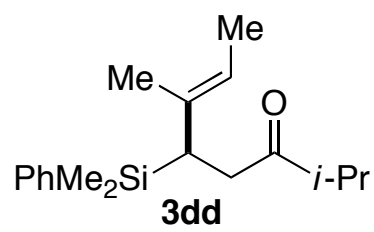

Entry 8. Colorless oil. $98 \%$ yield. The ee was determined on a Daicel Chiralpak AS column with hexane : 2-propanol $=500: 1$, flow $=0.5 \mathrm{~mL} / \mathrm{min}$. Retention times: $8.6 \mathrm{~min}$ $\left[(S)\right.$-enantiomer], 12.9 min $\left[(R)\right.$-enantiomer]. $94 \%$ ee. $[\alpha]^{20}{ }_{\mathrm{D}}+53.7\left(c 0.99, \mathrm{CHCl}_{3}\right)$. The absolute configuration was assigned by analogy with entry 5 .

${ }^{1} \mathrm{H}$ NMR $\left(\mathrm{CDCl}_{3}\right): \delta$ 7.49-7.46 (m, 2H), 7.36-7.32 (m, 3H), $4.98\left(\mathrm{q},{ }^{3} J_{\mathrm{HH}}=6.7 \mathrm{~Hz}, 1 \mathrm{H}\right)$, $2.70\left(\mathrm{dd},{ }^{2} J_{\mathrm{HH}}=16.8 \mathrm{~Hz}\right.$ and $\left.{ }^{3} J_{\mathrm{HH}}=11.2 \mathrm{~Hz}, 1 \mathrm{H}\right), 2.49\left(\mathrm{sept},{ }^{3} J_{\mathrm{HH}}=7.0 \mathrm{~Hz}, 1 \mathrm{H}\right), 2.38(\mathrm{dd}$, ${ }^{2} J_{\mathrm{HH}}=16.9 \mathrm{~Hz}$ and $\left.{ }^{3} J_{\mathrm{HH}}=4.1 \mathrm{~Hz}, 1 \mathrm{H}\right), 2.26\left(\mathrm{dd},{ }^{3} J_{\mathrm{HH}}=11.2\right.$ and $\left.4.1 \mathrm{~Hz}, 1 \mathrm{H}\right), 1.52\left(\mathrm{~d},{ }^{3} J_{\mathrm{HH}}=\right.$ $6.8 \mathrm{~Hz}, 3 \mathrm{H}), 1.50\left(\mathrm{~d},{ }^{4} J_{\mathrm{HH}}=1.0 \mathrm{~Hz}, 3 \mathrm{H}\right), 0.98\left(\mathrm{~d},{ }^{3} J_{\mathrm{HH}}=6.8 \mathrm{~Hz}, 3 \mathrm{H}\right), 0.97\left(\mathrm{~d},{ }^{3} J_{\mathrm{HH}}=6.8 \mathrm{~Hz}\right.$, $3 \mathrm{H}), 0.29$ (s, 3H), 0.27 (s, 3H). ${ }^{13} \mathrm{C} \mathrm{NMR}\left(\mathrm{CDCl}_{3}\right): \delta 214.5,138.1,136.3,134.1,129.2,127.8$, 117.5, 40.6, 40.4, 32.8, 18.4, 18.1, 18.0, 13.6, -3.5, -4.5. Anal. Calcd for $\mathrm{C}_{18} \mathrm{H}_{28} \mathrm{OSi}$ : C, 74.94; H, 9.78. Found: C, 75.11; H, 10.04. 


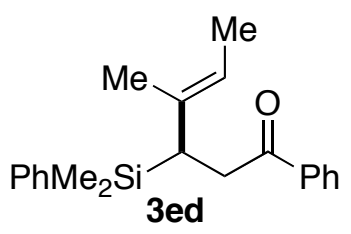

Entry 9. Colorless oil. 92\% yield. The ee was determined on a Daicel Chiralpak AD-H column with hexane : 2-propanol $=100: 1$, flow $=0.5 \mathrm{~mL} / \mathrm{min}$. Retention times: $12.7 \mathrm{~min}$ $\left[(S)\right.$-enantiomer], 14.1 min $\left[(R)\right.$-enantiomer]. $91 \%$ ee. $[\alpha]^{20}{ }_{\mathrm{D}}+44.3\left(c 1.03, \mathrm{CHCl}_{3}\right)$. The absolute configuration was assigned by analogy with entry 5 .

${ }^{1} \mathrm{H}$ NMR $\left(\mathrm{CDCl}_{3}\right): \delta$ 7.78-7.75 (m, 2H), 7.54-7.48 (m, 3H), 7.40-7.34 (m, 5H), 4.98 (q, $\left.{ }^{3} J_{\mathrm{HH}}=6.6 \mathrm{~Hz}, 1 \mathrm{H}\right), 3.18\left(\mathrm{dd},{ }^{2} J_{\mathrm{HH}}=16.4 \mathrm{~Hz}\right.$ and $\left.{ }^{3} J_{\mathrm{HH}}=11.1 \mathrm{~Hz}, 1 \mathrm{H}\right), 2.91\left(\mathrm{dd},{ }^{2} J_{\mathrm{HH}}=16.3\right.$ $\mathrm{Hz}$ and $\left.{ }^{3} J_{\mathrm{HH}}=4.0 \mathrm{~Hz}, 1 \mathrm{H}\right), 2.38\left(\mathrm{dd},{ }^{3} J_{\mathrm{HH}}=11.2\right.$ and $\left.3.9 \mathrm{~Hz}, 1 \mathrm{H}\right), 1.52\left(\mathrm{~d},{ }^{4} J_{\mathrm{HH}}=1.2 \mathrm{~Hz}, 3 \mathrm{H}\right)$, $1.50\left(\mathrm{~d},{ }^{3} J_{\mathrm{HH}}=7.1 \mathrm{~Hz}, 3 \mathrm{H}\right), 0.36(\mathrm{~s}, 3 \mathrm{H}), 0.30(\mathrm{~s}, 3 \mathrm{H}) .{ }^{13} \mathrm{C} \mathrm{NMR}\left(\mathrm{CDCl}_{3}\right): \delta 200.3,138.1$, 137.4, 135.8, 134.2, 132.7, 129.2, 128.6, 128.1, 127.9, 117.9, 38.4, 33.8, 17.7, 13.6, -3.4, -4.5. HRMS (ESI-TOF) calcd for $\mathrm{C}_{21} \mathrm{H}_{26} \mathrm{OSiNa}\left(\mathrm{M}+\mathrm{Na}^{+}\right) 345.1645$, found 345.1642.

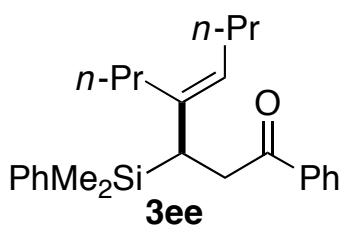

Entry 10. Colorless oil. $92 \%$ yield. The ee was determined on a Daicel Chiralcel OD-H column with hexane : 2-propanol $=98: 2$, flow $=0.2 \mathrm{~mL} / \mathrm{min}$. Retention times: $58.4 \mathrm{~min}$ $\left[(R)\right.$-enantiomer], 62.5 min $\left[(S)\right.$-enantiomer]. $93 \%$ ee. $[\alpha]^{20}{ }_{\mathrm{D}}+54.0\left(c 1.02, \mathrm{CHCl}_{3}\right)$. The absolute configuration was assigned by analogy with entry 5 .

${ }^{1} \mathrm{H} \mathrm{NMR}\left(\mathrm{CDCl}_{3}\right): \delta 7.76\left(\mathrm{~d},{ }^{3} J_{\mathrm{HH}}=7.0 \mathrm{~Hz}, 2 \mathrm{H}\right), 7.54-7.51(\mathrm{~m}, 2 \mathrm{H}), 7.50\left(\mathrm{t},{ }^{3} J_{\mathrm{HH}}=7.3 \mathrm{~Hz}\right.$, $1 \mathrm{H}), 7.38\left(\mathrm{t},{ }^{3} J_{\mathrm{HH}}=7.6 \mathrm{~Hz}, 2 \mathrm{H}\right), 7.37-7.33(\mathrm{~m}, 3 \mathrm{H}), 4.93\left(\mathrm{t},{ }^{3} J_{\mathrm{HH}}=7.3 \mathrm{~Hz}, 1 \mathrm{H}\right), 3.19\left(\mathrm{dd},{ }^{2} J_{\mathrm{HH}}\right.$ $=16.2 \mathrm{~Hz}$ and $\left.{ }^{3} J_{\mathrm{HH}}=10.5 \mathrm{~Hz}, 1 \mathrm{H}\right), 2.90\left(\mathrm{dd},{ }^{2} J_{\mathrm{HH}}=16.2 \mathrm{~Hz}\right.$ and $\left.{ }^{3} J_{\mathrm{HH}}=4.6 \mathrm{~Hz}, 1 \mathrm{H}\right), 2.39(\mathrm{dd}$, ${ }^{3} J_{\mathrm{HH}}=10.3$ and $\left.4.6 \mathrm{~Hz}, 1 \mathrm{H}\right), 2.07\left(\mathrm{ddd},{ }^{2} J_{\mathrm{HH}}=13.1 \mathrm{~Hz}\right.$ and ${ }^{3} J_{\mathrm{HH}}=10.0$ and $\left.6.7 \mathrm{~Hz}, 1 \mathrm{H}\right)$, 2.00-1.93 (m, 1H), 1.90-1.83 (m, 1H), $1.48\left(\mathrm{ddd},{ }^{2} J_{\mathrm{HH}}=13.4 \mathrm{~Hz}\right.$ and ${ }^{3} J_{\mathrm{HH}}=10.0$ and $4.8 \mathrm{~Hz}$, $1 \mathrm{H}), 1.40-1.31(\mathrm{~m}, 1 \mathrm{H}), 1.30-1.23(\mathrm{~m}, 2 \mathrm{H}), 1.16-1.06(\mathrm{~m}, 1 \mathrm{H}), 0.84\left(\mathrm{t},{ }^{3} J_{\mathrm{HH}}=7.4 \mathrm{~Hz}, 3 \mathrm{H}\right)$, $0.76\left(\mathrm{t},{ }^{3} J_{\mathrm{HH}}=7.3 \mathrm{~Hz}, 3 \mathrm{H}\right), 0.34(\mathrm{~s}, 3 \mathrm{H}), 0.31(\mathrm{~s}, 3 \mathrm{H}) .{ }^{13} \mathrm{C} \mathrm{NMR}\left(\mathrm{CDCl}_{3}\right): \delta 200.4,139.9$, 138.0, 137.7, 134.3, 132.7, 129.2, 128.5, 128.2, 127.8, 124.0, 39.2, 35.9, 30.8, 30.3, 23.5, 21.5, 14.5, 14.1, -3.3, -4.6. Anal. Calcd for $\mathrm{C}_{25} \mathrm{H}_{34} \mathrm{OSi}$ : C, 79.31; H, 9.05. Found: C, 79.05; $\mathrm{H}, 8.93$.

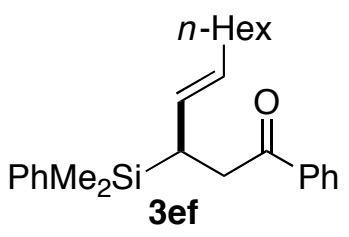

Entry 11. Colorless oil. $91 \%$ yield. The ee was determined on a Daicel Chiralpak AS column with hexane : 2-propanol $=98: 2$, flow $=0.2 \mathrm{~mL} / \mathrm{min}$. Retention times: $28.4 \mathrm{~min}$ $[(S)$-enantiomer $], 33.0$ min $[(R)$-enantiomer $] .56 \%$ ee. $[\alpha]^{20}{ }_{\mathrm{D}}+4.7\left(c 1.06, \mathrm{CHCl}_{3}\right)$. The absolute configuration was assigned by analogy with entry 5 .

${ }^{1} \mathrm{H} \mathrm{NMR}\left(\mathrm{CDCl}_{3}\right): \delta 7.78\left(\mathrm{~d},{ }^{3} J_{\mathrm{HH}}=7.3 \mathrm{~Hz}, 2 \mathrm{H}\right), 7.56-7.51(\mathrm{~m}, 2 \mathrm{H}), 7.51\left(\mathrm{t},{ }^{3} J_{\mathrm{HH}}=7.3 \mathrm{~Hz}\right.$, $1 \mathrm{H}), 7.40\left(\mathrm{t},{ }^{3} J_{\mathrm{HH}}=7.6 \mathrm{~Hz}, 2 \mathrm{H}\right), 7.38-7.35(\mathrm{~m}, 3 \mathrm{H}), 5.26\left(\mathrm{ddt},{ }^{3} J_{\mathrm{HH}}=15.3\right.$ and $8.8 \mathrm{~Hz}$ and $\left.{ }^{4} J_{\mathrm{HH}}=1.1 \mathrm{~Hz}, 1 \mathrm{H}\right), 5.16\left(\mathrm{dtd},{ }^{3} J_{\mathrm{HH}}=15.2 \mathrm{~Hz}\right.$ and $6.7 \mathrm{~Hz}$ and $\left.{ }^{4} J_{\mathrm{HH}}=0.7 \mathrm{~Hz}, 1 \mathrm{H}\right), 2.95(\mathrm{dd}$, ${ }^{2} J_{\mathrm{HH}}=15.7 \mathrm{~Hz}$ and $\left.{ }^{3} J_{\mathrm{HH}}=10.1 \mathrm{~Hz}, 1 \mathrm{H}\right), 2.89\left(\mathrm{dd},{ }^{2} J_{\mathrm{HH}}=15.7 \mathrm{~Hz}\right.$ and $\left.{ }^{3} J_{\mathrm{HH}}=4.2 \mathrm{~Hz}, 1 \mathrm{H}\right)$, 
$2.38\left(\mathrm{td},{ }^{3} J_{\mathrm{HH}}=9.3\right.$ and $\left.4.4 \mathrm{~Hz}, 1 \mathrm{H}\right), 1.90\left(\mathrm{q},{ }^{3} J_{\mathrm{HH}}=6.6 \mathrm{~Hz}, 2 \mathrm{H}\right), 1.30-1.18(\mathrm{~m}, 8 \mathrm{H}), 0.86(\mathrm{t}$, $\left.{ }^{3} J_{\mathrm{HH}}=7.1 \mathrm{~Hz}, 3 \mathrm{H}\right), 0.33(\mathrm{~s}, 3 \mathrm{H}), 0.31(\mathrm{~s}, 3 \mathrm{H}) .{ }^{13} \mathrm{C} \mathrm{NMR}\left(\mathrm{CDCl}_{3}\right): \delta 200.2,137.4,134.2$, 132.8, 129.9, 129.3, 129.1, 128.6, 128.2, 127.9, 38.4, 32.9, 31.9, 29.9, 28.8, 28.4, 22.8, 14.2, -4.1, -5.1. Anal. Calcd for $\mathrm{C}_{25} \mathrm{H}_{34} \mathrm{OSi}$ : C, 79.31; H, 9.05. Found: C, 79.46; H, 9.08.

\section{Procedure for Scheme 1.}<smiles>C=C(C)C(CC(=O)c1ccccc1)[SiH2]c1ccccc1</smiles>

A solution of $\mathrm{RhCl}\left(\mathrm{PPh}_{3}\right)_{3}(13.5 \mathrm{mg}, 14.6 \mu \mathrm{mol})$ and 3ea $(87.1 \mathrm{mg}, 0.282 \mathrm{mmol}$; Table 2, entry 5) in $\mathrm{MeOH}(2.0 \mathrm{~mL})$ was purged with $\mathrm{H}_{2}$ gas with a balloon and was stirred for $6 \mathrm{~h}$ at room temperature under $\mathrm{H}_{2}$. The reaction mixture was directly passed through a pad of silica gel with EtOAc and the solvent was removed under vacuum. The residue was purified by silica gel preparative TLC with hexane/EtOAc $=20 / 1$ to afford compound $\mathbf{4}$ as a colorless oil (81.0 mg, $0.261 \mathrm{mmol} ; 92 \%$ yield).

${ }^{1} \mathrm{H} \mathrm{NMR}\left(\mathrm{CDCl}_{3}\right): \delta 7.79\left(\mathrm{~d},{ }^{3} J_{\mathrm{HH}}=7.3 \mathrm{~Hz}, 2 \mathrm{H}\right), 7.56-7.53(\mathrm{~m}, 2 \mathrm{H}), 7.51\left(\mathrm{t},{ }^{3} J_{\mathrm{HH}}=7.5 \mathrm{~Hz}\right.$, $1 \mathrm{H}), 7.39\left(\mathrm{t},{ }^{3} J_{\mathrm{HH}}=7.8 \mathrm{~Hz}, 2 \mathrm{H}\right), 7.34-7.30(\mathrm{~m}, 3 \mathrm{H}), 2.99\left(\mathrm{dd},{ }^{2} J_{\mathrm{HH}}=17.1 \mathrm{~Hz}\right.$ and ${ }^{3} J_{\mathrm{HH}}=7.5$ $\mathrm{Hz}, 1 \mathrm{H}), 2.92\left(\mathrm{dd},{ }^{2} J_{\mathrm{HH}}=17.1 \mathrm{~Hz}\right.$ and $\left.{ }^{3} J_{\mathrm{HH}}=5.2 \mathrm{~Hz}, 1 \mathrm{H}\right), 1.94\left(\right.$ sept of d, ${ }^{3} J_{\mathrm{HH}}=6.7$ and 4.1 $\mathrm{Hz}, 1 \mathrm{H}), 1.79\left(\mathrm{ddd},{ }^{3} J_{\mathrm{HH}}=7.5,5.2\right.$, and $\left.4.1 \mathrm{~Hz}, 1 \mathrm{H}\right), 0.91\left(\mathrm{~d},{ }^{3} J_{\mathrm{HH}}=6.7 \mathrm{~Hz}, 3 \mathrm{H}\right), 0.86(\mathrm{~d}$, $\left.{ }^{3} J_{\mathrm{HH}}=6.9 \mathrm{~Hz}, 3 \mathrm{H}\right), 0.37(\mathrm{~s}, 3 \mathrm{H}), 0.34(\mathrm{~s}, 3 \mathrm{H}) .{ }^{13} \mathrm{C} \mathrm{NMR}\left(\mathrm{CDCl}_{3}\right): \delta 201.1,139.2,137.3$, 134.1, 132.8, 129.0, 128.6, 128.1, 127.9, 36.3, 29.0, 28.3, 23.1, 21.6, -2.0, -2.7.<smiles>CC(C)C(C)C(C)C(CC(=O)c1ccccc1)C(C)C</smiles>

The reaction was conducted according to the literature procedure. ${ }^{10} \mathrm{HBF}_{4}{ }^{\bullet} \mathrm{OEt}_{2}(88.0 \mu \mathrm{L}$, $0.647 \mathrm{mmol})$ was added to a solution of compound $4(81.0 \mathrm{mg}, 0.261 \mathrm{mmol})$ in $\mathrm{CH}_{2} \mathrm{Cl}_{2}(1.6$ $\mathrm{mL}$ ) at $0{ }^{\circ} \mathrm{C}$ and the mixture was stirred for $1 \mathrm{~h}$ at $0{ }^{\circ} \mathrm{C}$. The volatiles were removed under vacuum and $\mathrm{MeOH}(1.6 \mathrm{~mL})$ and $\mathrm{THF}(1.6 \mathrm{~mL})$ were added to the residue. The solution was cooled to $0{ }^{\circ} \mathrm{C}$ and $\mathrm{KF}(32.0 \mathrm{mg}, 0.551 \mathrm{mmol})$ and $\mathrm{KHCO}_{3}(148 \mathrm{mg}, 1.48 \mathrm{mmol})$ were added. The resulting mixture was stirred for $15 \mathrm{~min}$ at $0{ }^{\circ} \mathrm{C}$ and $\mathrm{H}_{2} \mathrm{O}_{2}(390 \mu \mathrm{L}, 3.82 \mathrm{mmol} ; 30 \mathrm{wt} \%$ aqueous) was then added to it. After stirring for $9 \mathrm{~h}$ at $0{ }^{\circ} \mathrm{C}$, the reaction was quenched with saturated $\mathrm{Na}_{2} \mathrm{SO}_{3}$ aqueous and diluted with $1 \mathrm{M} \mathrm{HCl}$ aqueous. This was extracted with $\mathrm{Et}_{2} \mathrm{O}$ and the organic layer was dried over $\mathrm{MgSO}_{4}$, filtered, and concentrated under vacuum. The residue was chromatographed on silica gel with hexane/EtOAc $=10 / 1$ to afford compound 5 as a colorless oil $(44.5 \mathrm{mg}, 0.231 \mathrm{mmol} ; 89 \%$ yield $)$.

$[\alpha]^{20}{ }_{\mathrm{D}}+78.0\left(c 1.00, \mathrm{CHCl}_{3}\right)$. The absolute configuration was determined by comparison of the optical rotation with that reported in the literature. ${ }^{9}$

${ }^{1} \mathrm{H} \mathrm{NMR}\left(\mathrm{CDCl}_{3}\right): \delta 7.96\left(\mathrm{~d},{ }^{3} J_{\mathrm{HH}}=7.2 \mathrm{~Hz}, 2 \mathrm{H}\right), 7.58\left(\mathrm{t},{ }^{3} J_{\mathrm{HH}}=7.4 \mathrm{~Hz}, 1 \mathrm{H}\right), 7.47\left(\mathrm{t},{ }^{3} J_{\mathrm{HH}}\right.$ $=7.6 \mathrm{~Hz}, 2 \mathrm{H}), 4.02-3.97(\mathrm{~m}, 1 \mathrm{H}), 3.19(\mathrm{~s}, 1 \mathrm{H}), 3.17\left(\mathrm{dd},{ }^{2} J_{\mathrm{HH}}=17.4 \mathrm{~Hz}\right.$ and ${ }^{3} J_{\mathrm{HH}}=2.3 \mathrm{~Hz}$, $1 \mathrm{H}) 3.03\left(\mathrm{dd},{ }^{2} J_{\mathrm{HH}}=17.4 \mathrm{~Hz}\right.$ and $\left.{ }^{3} J_{\mathrm{HH}}=9.6 \mathrm{~Hz}, 1 \mathrm{H}\right), 1.81\left(\right.$ sept of d, ${ }^{3} J_{\mathrm{HH}}=6.6$ and $5.8 \mathrm{~Hz}$, $1 \mathrm{H}), 1.01\left(\mathrm{~d},{ }^{3} J_{\mathrm{HH}}=6.8 \mathrm{~Hz}, 3 \mathrm{H}\right), 0.99\left(\mathrm{~d},{ }^{3} J_{\mathrm{HH}}=6.8 \mathrm{~Hz}, 3 \mathrm{H}\right) \cdot{ }^{13} \mathrm{C} \mathrm{NMR}\left(\mathrm{CDCl}_{3}\right): \delta 201.5$, $137.1,133.6,128.8,128.2,72.5,42.1,33.3,18.7,18.0$.

${ }^{10}$ Matsumoto, Y.; Hayashi, T.; Ito, Y. Tetrahedron 1994, 50, 335. 


\section{General Procedure for Equations 1 and 2.}

A solution of $\mathrm{TiCl}_{4}\left(0.15 \mathrm{~mL}, 0.15 \mathrm{mmol} ; 1.0 \mathrm{M}\right.$ in $\left.\mathrm{CH}_{2} \mathrm{Cl}_{2}\right)$ was added to a solution of compound 3dc or 3dd $(0.15 \mathrm{mmol})$ in $\mathrm{CH}_{2} \mathrm{Cl}_{2}(1.4 \mathrm{~mL})$ at $-78{ }^{\circ} \mathrm{C}$. After stirring for $5 \mathrm{~min}$ at $-78{ }^{\circ} \mathrm{C}$, the reaction was quenched with saturated $\mathrm{NaCl}$ aqueous. The mixture was warmed to room temperature and it was extracted with $\mathrm{CH}_{2} \mathrm{Cl}_{2}$. The organic layer was dried over $\mathrm{MgSO}_{4}$, filtered, and concentrated under vacuum. The residue was chromatographed on silica gel with hexane/EtOAc to afford compound $\mathbf{6}$ or $\mathbf{7 .}$



Equation 1. Colorless oil. $89 \%$ yield. The ee was determined on a Daicel Chiralpak AD-H column with hexane : 2-propanol $=90: 10$, flow $=0.5 \mathrm{~mL} / \mathrm{min}$. Retention times: $14.7 \mathrm{~min}$ [major enantiomer], $19.1 \mathrm{~min}$ [minor enantiomer]. $92 \%$ ee. $[\alpha]^{20}{ }_{\mathrm{D}}+114\left(c 1.10, \mathrm{CHCl}_{3}\right)$. The relative and absolute configurations were assigned by analogy with the literature examples. ${ }^{11}$

${ }^{1} \mathrm{H}$ NMR $\left(\mathrm{CDCl}_{3}\right): \delta 5.19(\mathrm{~s}, 1 \mathrm{H}), 2.44-2.40(\mathrm{~m}, 1 \mathrm{H}), 2.35\left(\mathrm{~d},{ }^{2} J_{\mathrm{HH}}=16.3 \mathrm{~Hz}, 1 \mathrm{H}\right), 2.27$ $\left(\mathrm{dt},{ }^{2} J_{\mathrm{HH}}=16.6 \mathrm{~Hz}\right.$ and $\left.J_{\mathrm{HH}}=2.5 \mathrm{~Hz}, 1 \mathrm{H}\right), 2.14\left(\mathrm{dd},{ }^{2} J_{\mathrm{HH}}=12.5 \mathrm{~Hz}\right.$ and $\left.{ }^{3} J_{\mathrm{HH}}=4.0 \mathrm{~Hz}, 1 \mathrm{H}\right)$, 2.08-2.00 (m, 1H), 1.93-1.87 (m, 1H), 1.87-1.81 (m, 1H), 1.81-1.75 (m, 1H), $1.74\left(\mathrm{sept},{ }^{3} J_{\mathrm{HH}}\right.$ $=6.8 \mathrm{~Hz}, 1 \mathrm{H}), 1.51(\mathrm{~s}, 1 \mathrm{H}), 1.37\left(\mathrm{qt}, J_{\mathrm{HH}}=13.2 \mathrm{~Hz}\right.$ and $\left.{ }^{3} J_{\mathrm{HH}}=3.4 \mathrm{~Hz}, 1 \mathrm{H}\right), 1.18\left(\mathrm{qt}, J_{\mathrm{HH}}=\right.$ $12.7 \mathrm{~Hz}$ and $\left.{ }^{3} J_{\mathrm{HH}}=4.0 \mathrm{~Hz}, 1 \mathrm{H}\right), 0.98\left(\mathrm{~d},{ }^{3} J_{\mathrm{HH}}=6.6 \mathrm{~Hz}, 3 \mathrm{H}\right), 0.95\left(\mathrm{~d},{ }^{3} J_{\mathrm{HH}}=6.6 \mathrm{~Hz}, 3 \mathrm{H}\right)$, 0.97-0.89 (m, 1H). ${ }^{13} \mathrm{C} \mathrm{NMR}\left(\mathrm{CDCl}_{3}\right): \delta 148.0,116.8,84.8,58.7,45.4,32.2,30.6,30.2,29.4$, 26.5, 17.8, 17.3. Anal. Calcd for $\mathrm{C}_{12} \mathrm{H}_{20} \mathrm{O}$ : C, 79.94; H, 11.18. Found: C, 80.21; H, 11.46.

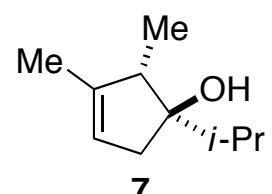

Equation 2. Colorless oil. $75 \%$ yield. The ee was determined on a Daicel Chiralpak AS column with hexane : 2-propanol $=500: 1$, flow $=0.5 \mathrm{~mL} / \mathrm{min}$. Retention times: $12.6 \mathrm{~min}$ [major enantiomer], $13.7 \mathrm{~min}$ [minor enantiomer]. 93\% ee. $[\alpha]^{20}{ }_{\mathrm{D}}+66.4\left(c 0.70, \mathrm{CHCl}_{3}\right)$. The relative and absolute configurations were assigned by analogy with the literature examples. ${ }^{11}$

${ }^{1} \mathrm{H} \mathrm{NMR}\left(\mathrm{CDCl}_{3}\right): \delta 5.26(\mathrm{~s}, 1 \mathrm{H}), 2.34\left(\mathrm{~d},{ }^{2} J_{\mathrm{HH}}=16.4 \mathrm{~Hz}, 1 \mathrm{H}\right), 2.22\left(\mathrm{q},{ }^{3} J_{\mathrm{HH}}=6.9 \mathrm{~Hz}\right.$, $1 \mathrm{H}), 2.17\left(\mathrm{~d},{ }^{2} J_{\mathrm{HH}}=16.1 \mathrm{~Hz}, 1 \mathrm{H}\right), 1.83\left(\mathrm{sept},{ }^{3} J_{\mathrm{HH}}=6.7 \mathrm{~Hz}, 1 \mathrm{H}\right), 1.74(\mathrm{~s}, 3 \mathrm{H}), 1.56(\mathrm{~s}, 1 \mathrm{H})$, $0.97\left(\mathrm{~d},{ }^{3} J_{\mathrm{HH}}=6.7 \mathrm{~Hz}, 3 \mathrm{H}\right), 0.95\left(\mathrm{~d},{ }^{3} J_{\mathrm{HH}}=6.7 \mathrm{~Hz}, 3 \mathrm{H}\right), 0.89\left(\mathrm{~d},{ }^{3} J_{\mathrm{HH}}=6.9 \mathrm{~Hz}, 3 \mathrm{H}\right) .{ }^{13} \mathrm{C}$ NMR $\left(\mathrm{CDCl}_{3}\right): \delta 145.4,120.9,85.9,53.8,44.0,31.9,17.3,17.2,15.6,14.4$. Anal. Calcd for $\mathrm{C}_{10} \mathrm{H}_{18} \mathrm{O}: \mathrm{C}, 77.87 ; \mathrm{H}, 11.76$. Found: $\mathrm{C}, 78.03 ; \mathrm{H}, 11.93$.

${ }^{11}$ (a) Masse, C. E.; Dakin, L. A.; Knight, B. S.; Panek, J. S. J. Org. Chem. 1997, 62, 9335. (b) Mikami, K.; Maeda, T.; Kishi, N.; Nakai, T. Tetrahedron Lett. 1984, 25, 5151. 
IV. ${ }^{1}$ H NMR Spectra
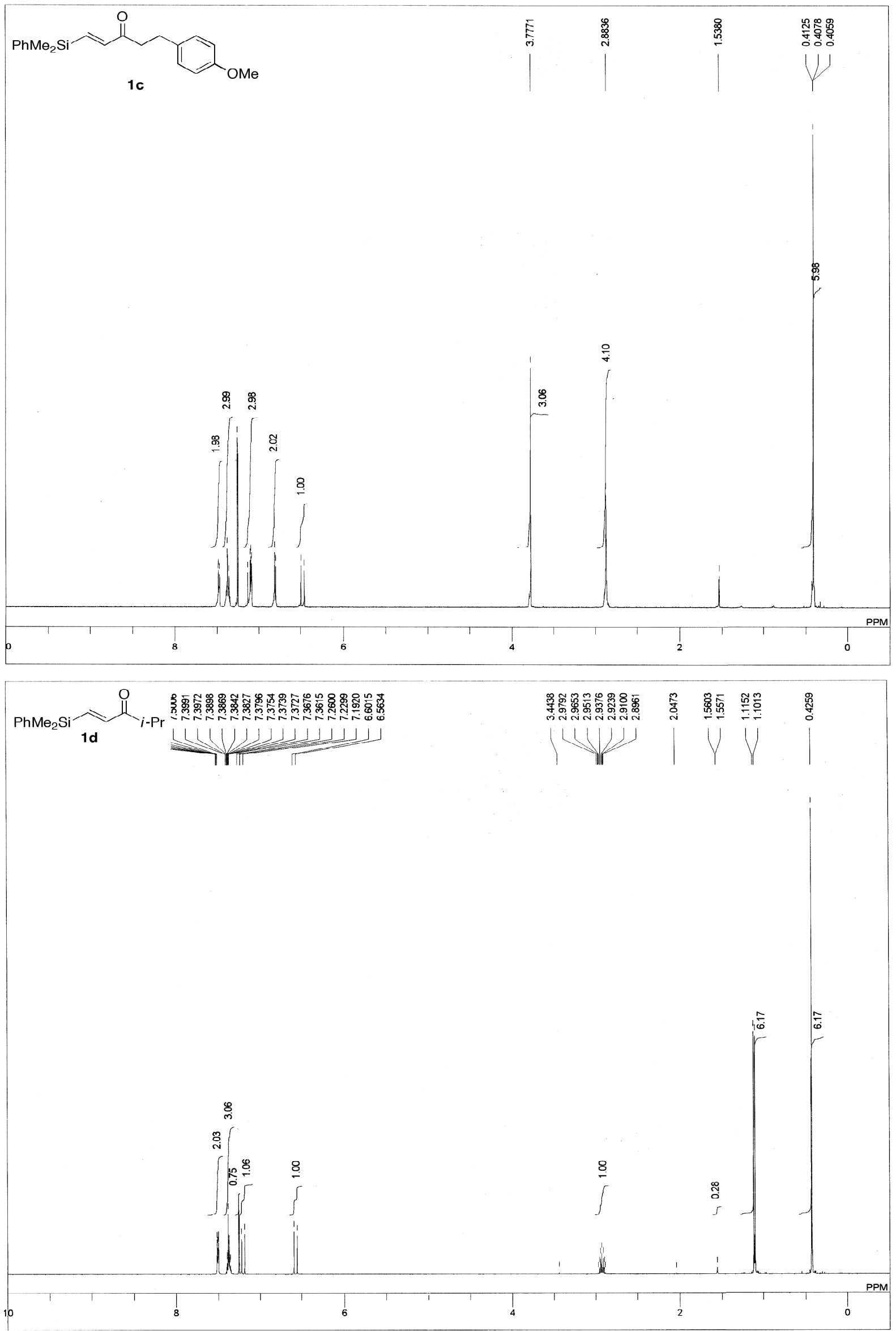





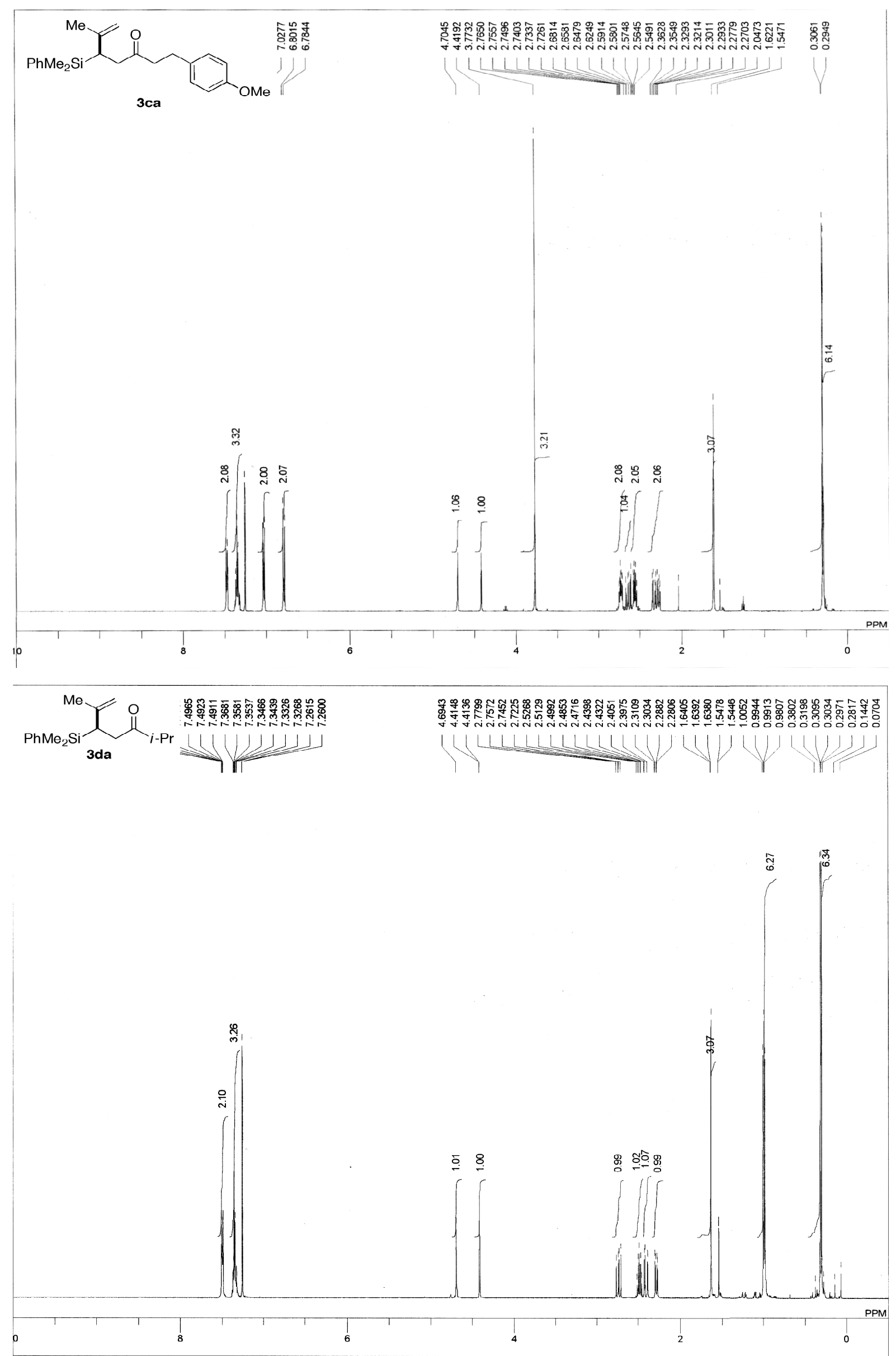

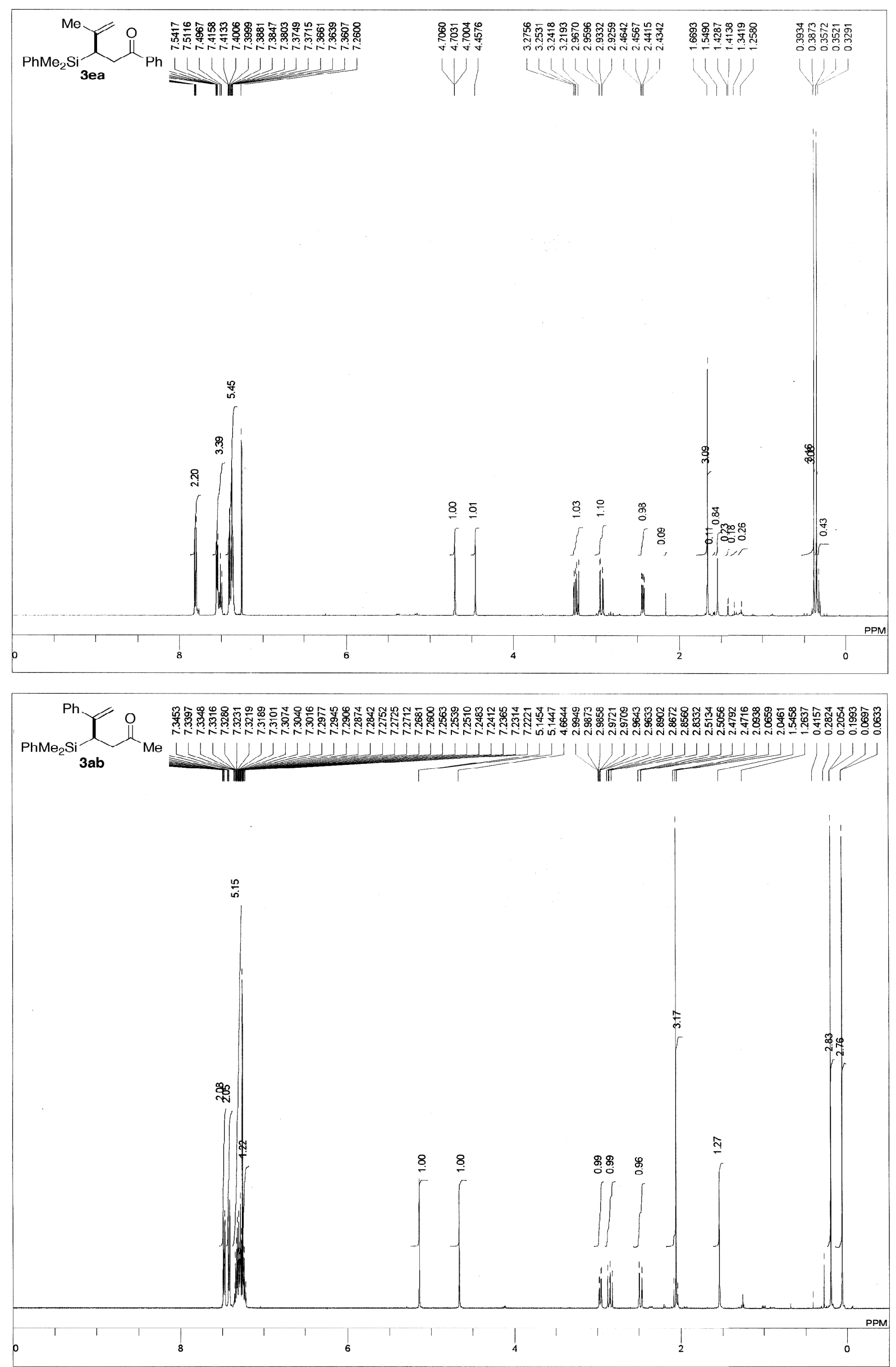

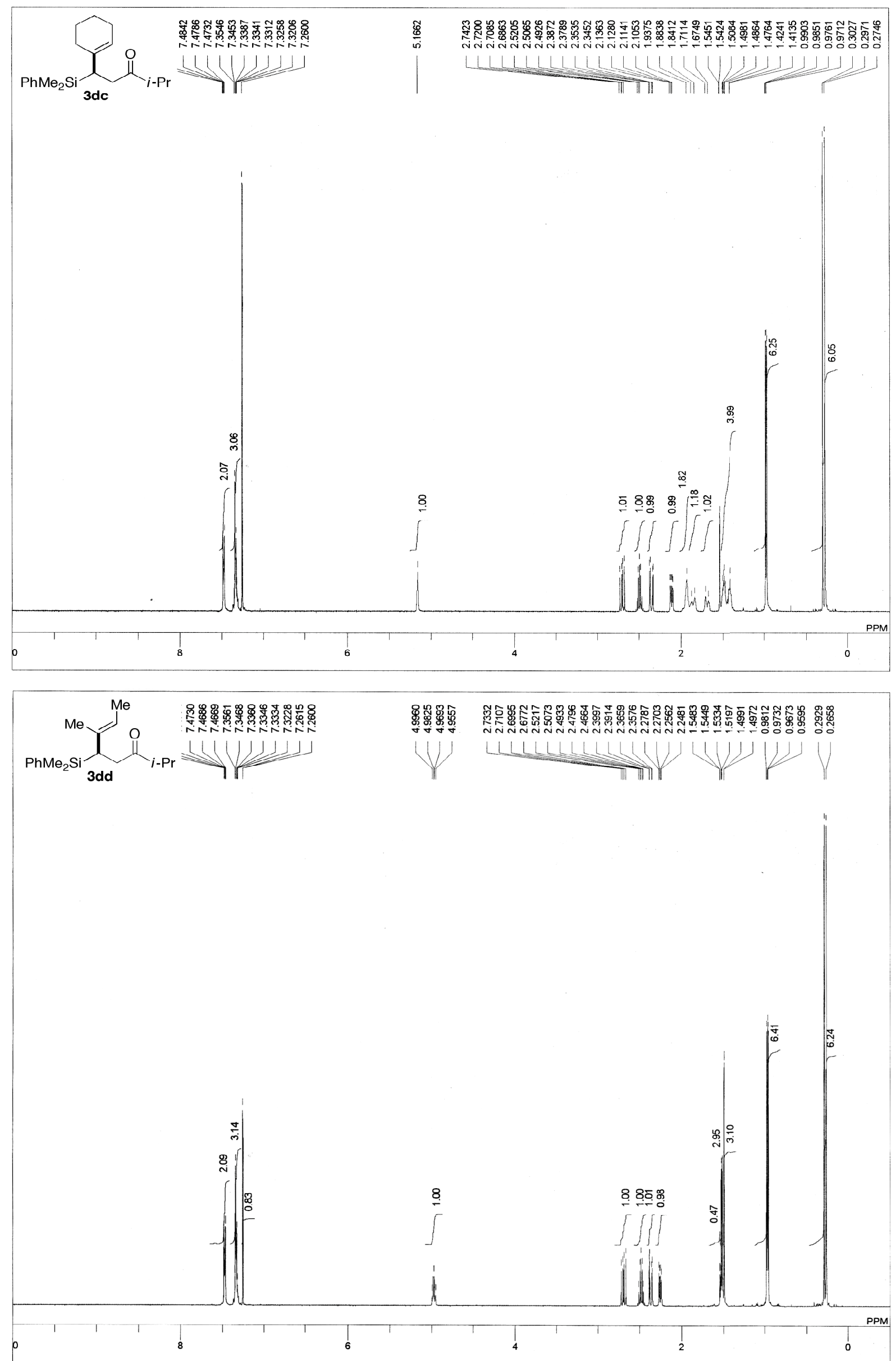

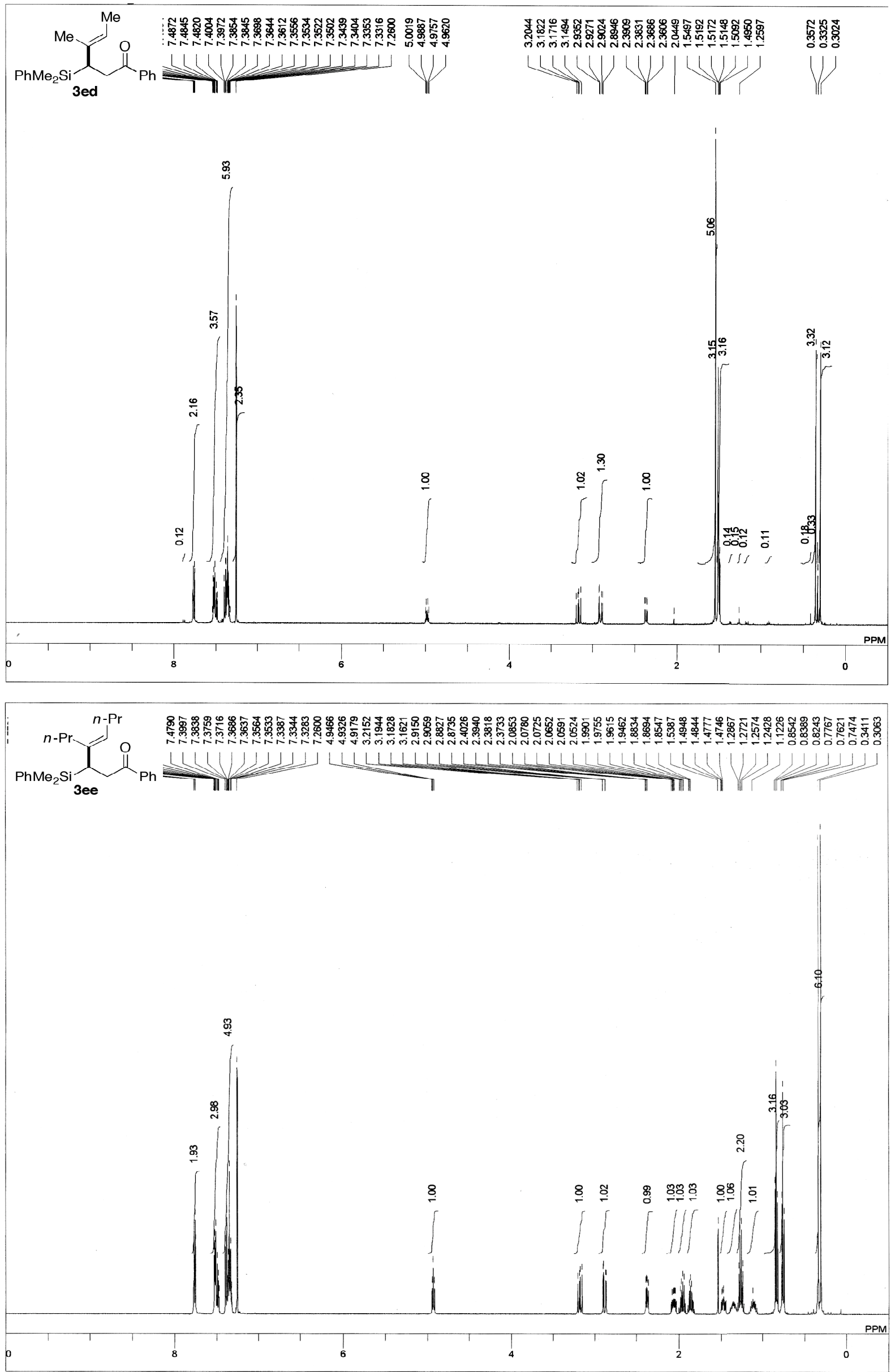

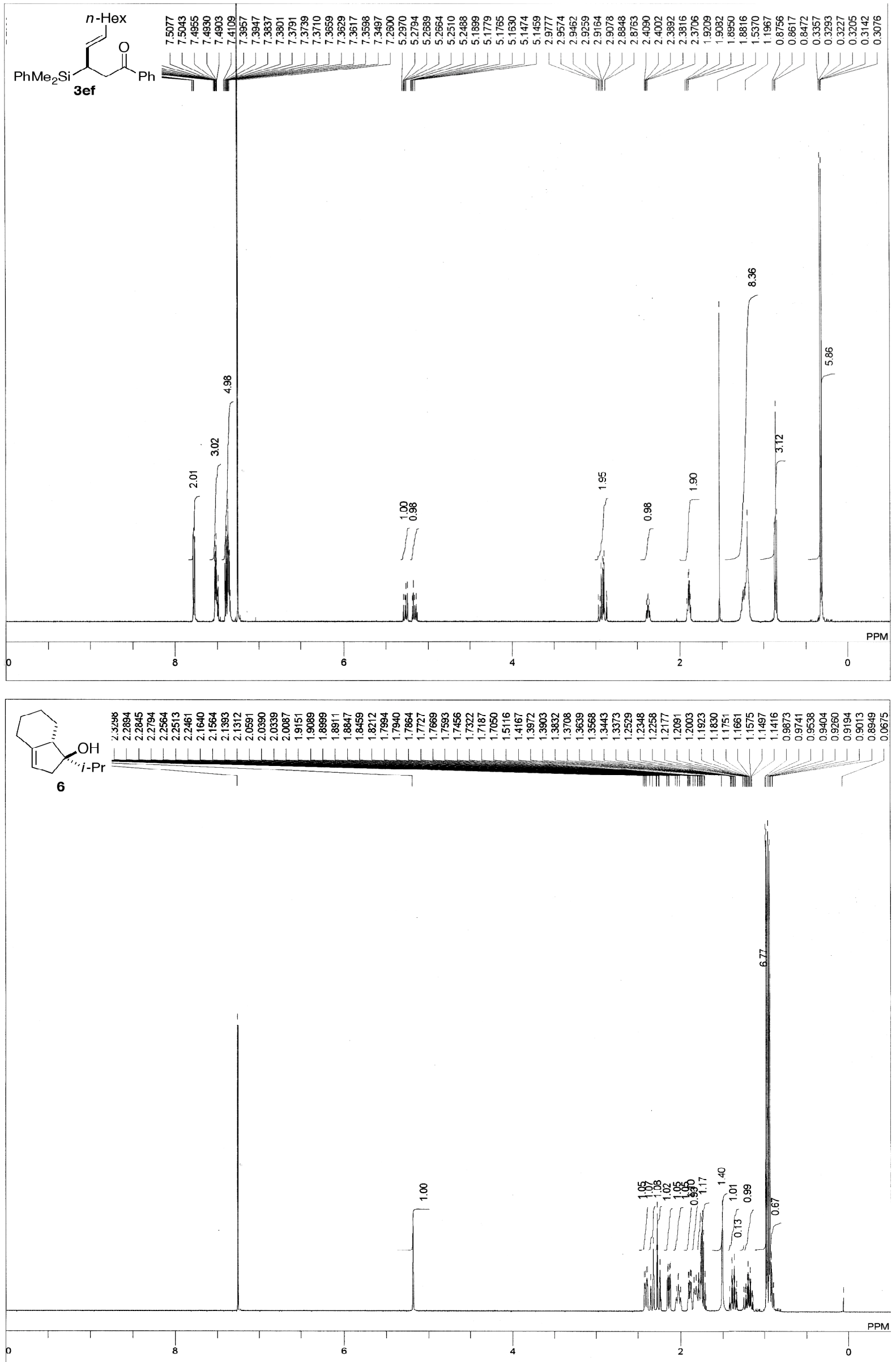


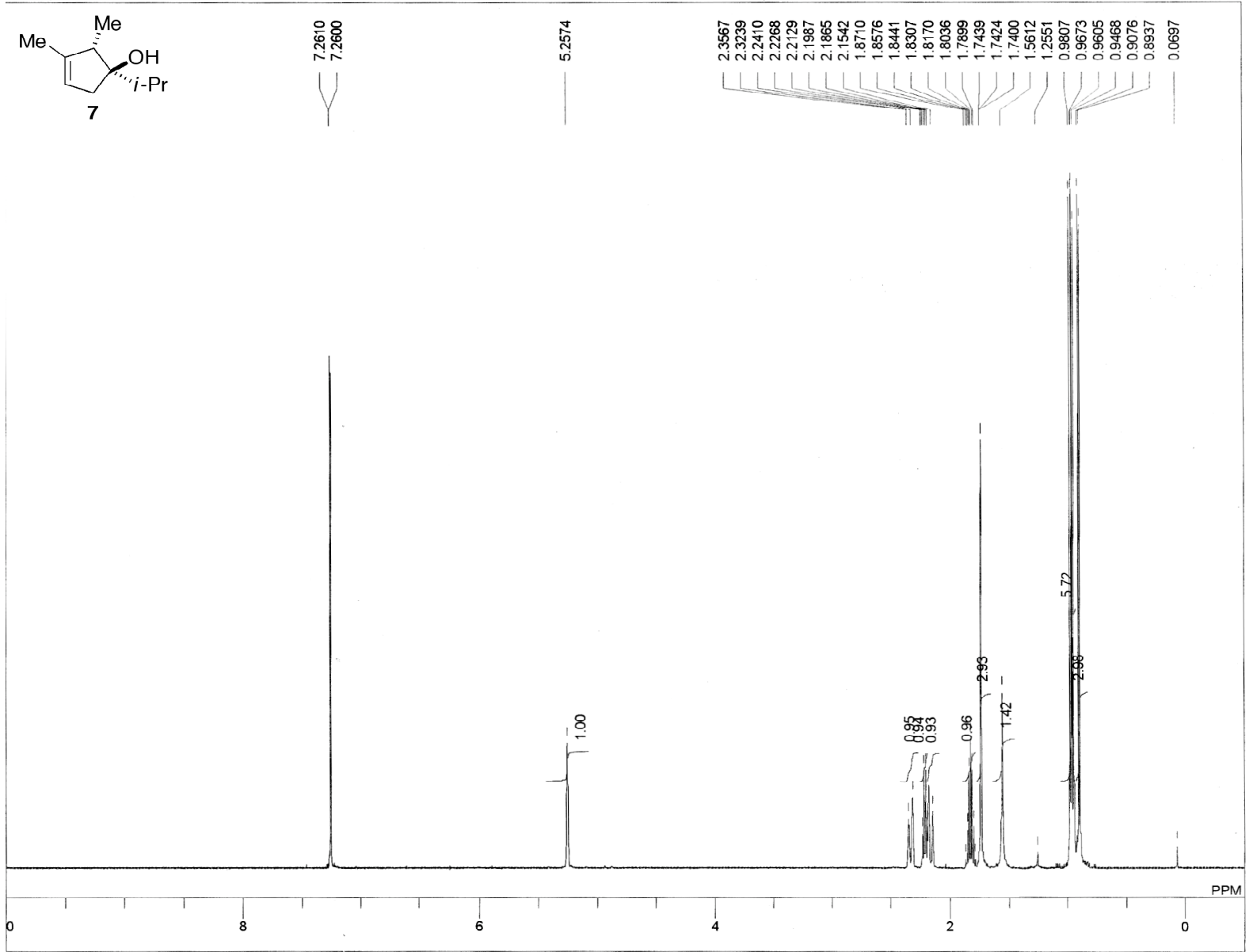

